\title{
Avaliação de fadiga e fatores associados em pessoas submetidas à hemodiálise
}

Evaluación de la fatiga y factores asociados en personas sometidas a hemodiálisis

Fatigue assessment and associated factors in people undergoing hemodialysis

Como citar este artigo:

Kickhöfel, Marinéia Albrecht; Schwartz, Eda; Spagnolo, Lílian Moura de Lima; Sampaio, Aurélia Danda; Cunha, Tuany Nunes; Lise, Fernanda. Avaliação de fadiga e fatores associados em pessoas submetidas à hemodiálise. Revista Cuidarte. 2020;12(3):e12120. http://dx.doi.org/10.15649/cuidarte.2120

Revista Cuidarte

Rev Cuid. Sep - Dic 2021; 12(3): e2120

doij http://dx.doi.org/10.15649/cuidarte.2120

E-ISSN: 2346-3414

(1) Marinéia Albrecht Kickhöfel ${ }^{1}$

(1) Eda Schwartz ${ }^{2}$

(1) Lílian Moura de Lima Spagnolo ${ }^{3}$

(1) Aurélia Danda Sampaio ${ }^{4}$

(1) Tuany Nunes Cunha ${ }^{5}$

(1) Fernanda Lise ${ }^{6}$

1 Universidade Federal de Pelotas, Pelotas, RS, Brasil, E-mail: marineiakickhofel@gmail.com Autora para correspondência

2 Universidade Federal de Pelotas, Pelotas, RS, Brasil.

E-mail: edaschwa@gmail.com

3 Universidade Federal de Pelotas, Pelotas, RS, Brasil.

E-mail: lima.lilian@gmail.com

4 Universidade Federal de Pelotas, Pelotas, RS, Brasil. E-mail: aurelia.sampaio@hotmail.com

5 Universidade Federal de Pelotas, Pelotas, RS, Brasil.

E-mail: tuanynunes@hotmail.com

6 Universidade Federal de Pelotas, Pelotas, RS, Brasil.

E-mail: fernandalise@gmail.com

\section{Resumo}

Introdução: o sintoma de fadiga é considerado incapacitante e afeta a qualidade de vida das pessoas com doença renal crônica, em tratamento de hemodiálise. Objetivo: avaliar a prevalência, intensidade e severidade do sintoma de fadiga em pessoas com doença renal crônica em tratamento hemodialítico. Materiais e Método: Estudo quantitativo descritivo, do qual participaram 335 pessoas em tratamento hemodialítico. Para a coleta dos dados foram utilizadas as escalas Visual Analog Scale" de 100mm, escala de severidade de fadiga e um questionário sociodemográfico. Foram analisadas as variáveis dependentes das escalas de fadiga, com as independentes de caracterização sociodemográfica e clínica. Resultados: Identificaram-se $58 \%$ do sexo masculino, idade entre 20-59 anos em 52,2\%, predomínio de cor branca em 68,2\%, $68,6 \%$ com baixa escolaridade, aposentadoria como principal renda em 55,7\%, renda entre $\mathrm{R} \$ 800,01$ e $\mathrm{R} \$ 1760,00$ em 42,4\%, a prevalência de fadiga foi de $63,0 \%$, fadiga severa em viúvos $(35,2 \%)$ e mulheres $(27,6 \%)$, sendo que a principal comorbidade foi a Hipertensão Arterial Sistêmica (40,9\%). A intensidade de fadiga teve o valor médio de $42,3(\mathrm{DP}=32,2)$ e a severidade de fadiga teve valor médio de 35,8(DP=17,8). Conclusões: evidenciou-se que o fator socioeconômico e as comorbidades influenciam no sintoma de fadiga em mulheres, viúvos e idosos. O uso de instrumentos de avaliação pode contribuir para melhorar a abordagem da Enfermagem, e em consequência, o bem-estar e a qualidade de vida destas pessoas.

Palavras chave: Insuficiência Renal Crônica; Fadiga; Diálise Renal; Enfermagem.

Recebido: 24 de fevereiro de 2021

Aceito: 02 de agosto de 2021

Publicado: 06 de outubro de 2021 $\square *$ Correspondencia

Marinéia Albrecht Kickhöfel

E-mail:marineiakickhofel@gmail.com 


\section{Fatigue assessment and associated factors in people undergoing hemodialysis}

\section{Abstract}

Introduction: The symptom of fatigue is considered incapacitating and affects the quality of life of people with chronic kidney disease, undergoing hemodialysis treatment. Objective: to assess the prevalence, intensity, and severity of the symptoms of fatigue in people with chronic kidney disease in hemodialysis treatment. Materials and Methods: Descriptive quantitative study, in which 335 in hemodialysis treatment participated. For the data collection, were used the 100mm Visual Analog Scale", the scale of severity of the fatigue, and a sociodemographic questionnaire. Were analyzed variable dependent on the fatigue scale, with the independent variables of sociodemographic and clinical characterization. Results: $58 \%$ were identified as male, aged between 20-59 years old in 52.2\%, predominance of White color in $68.2 \%, 68.6 \%$ with low schooling, retirement as main income in 55.7\%, yields between $\mathrm{R} \$ 800.01$ and $\mathrm{R} \$$ 1760.00 in $42.4 \%$, with a prevalence of fatigue were $63.0 \%$, severe fatigue in viúvos (35.2\%) and women (27.6\%), being that the main comorbidities of Systemic Arterial Hypertension (40.9\%). At the intensity of fatigue, it has a mean value of $42.3(\mathrm{DP}=32.2)$ and at the severity of fatigue, it has a mean value of $35.8(\mathrm{DP}=17.8)$. Conclusions: We evidenced that the socioeconomic factor in comorbidities influences the symptoms of fatigue in women, widowers, and the elderly. The use of assessment instruments can contribute to help us address the sickness, and consequently, or improve the quality of life of these people.

Key words: Renal Insufficiency, Chronic; Fatigue; Renal Dialysis; Nursing.

\section{Evaluación de la fatiga y factores asociados en personas sometidas a hemodiálisis}

\section{Resumen}

Introducción: el síntoma de la fatiga se considera incapacitante y afecta a la calidad de vida de las personas con enfermedad renal crónica en tratamiento de hemodiálisis. Objetivo: evaluar la prevalencia, intensidad y gravedad del síntoma fatiga en personas con enfermedad renal crónica en tratamiento de hemodiálisis. Materiales y métodos: Estudio descriptivo cuantitativo, en el que participaron 335 personas en tratamiento de hemodiálisis. Para la recogida de los datos se utilizó la Visual Analog Scale" de 100mm, escala de gravedad de la fatiga y un cuestionario sociodemográfico. Se analizaron las variables dependientes de las escalas de fatiga, con las independientes de caracterización sociodemográfica y clínica. Resultados: $58 \%$ eran hombres, 52,2\% tenían entre 20-59 años, 68,2\% eran predominantemente blancos, 68,6\% tenían bajo nivel de estudios, 55,7\% tenían la jubilación como ingreso principal, 42,4\% tenían ingresos entre $\mathrm{R} \$ 800,01$ y $\mathrm{R} \$ 1760,00$, la prevalencia de la fatiga fue de $63,0 \%$, fatiga severa en viudos (35,2\%) y mujeres (27,6\%), y la principal comorbilidad fue la hipertensión arterial sistémica (40,9\%). La intensidad de la fatiga tuvo un valor medio de 42,3 ( $D P=32,2)$ y la gravedad de la fatiga tuvo un valor medio de 35,8 ( $\mathrm{DP}=17,8)$. Conclusiones: se evidenció que el factor socioeconómico y las comorbilidades influyen en el síntoma de fatiga en mujeres, viudos y ancianos. El uso de instrumentos de evaluación puede contribuir a mejorar el abordaje de la enfermedad y, en consecuencia, el bienestar y la calidad de vida de las personas.

Palablas clave: Insuficiencia renal crónica; Fatiga; Diálisis renal; Enfermería. 


\section{Introdução}

A Doença Renal Crônica (DRC) é caracterizada por alterações heterogêneas ou anormalidades que afetam a estrutura e a função renal, com redução progressiva da filtração glomerular por, pelo menos, três meses'. Sua ocorrência é considerada um problema de saúde pública em todo o mundo. De acordo com o Censo Brasileiro de Diálise de 2018 a prevalência estimada é de 640 pacientes por milhão da população (pmp) em diálise, sendo a Hemodiálise (HD) a Terapia Renal Substitutiva (TRS) mais empregada em $92 \%$ dos usuários, predomínio do sexo masculino 58\%, faixa etária entre 45-64 anos (41,5\%), e com mais de 65 anos (35\%) 2 .

Durante o percurso da DRC, a fadiga é considerada um sintoma incapacitante, com diversos distúrbios clínicos e neurológicos, difícil de definir e estudar, pois trata de uma entidade distinta e altamente subjetiva ${ }^{3}$, tornando-se o principal sintoma de

A fadiga é considerada um sintoma incapacitante, com diversos distúrbios clínicos e neurológicos, difícil de definir e estudar, pois trata de uma entidade distinta e altamente subjetiva ${ }^{3}$. impacto negativo na qualidade de vida (QV). Além disso, por estar associada a comorbidades, tais como depressão, anemia, alterações na qualidade do sono, além de fatores demográficos, tornando alta sua prevalência, na maioria dos casos permanecendo subdiagnosticada e subtratada, aumentando os eventos cardiovasculares e mortalidade em pacientes com DRC4.

As pessoas com DRC que desenvolvem fadiga apresentam maior prevalência de xerodermia (pele seca), prurido (coceira), mialgia (dor muscular), artralgia (dor nas articulações), osteodinia (dor nos ossos), síndrome das pernas inquietas, dispneia (falta de ar), sensação de tristeza e ansiedade, dificuldade de concentração e para se excitar, em relação aos não fadigados, pode gerar limitações de ordem física, social e emocional, com sérias repercussões na vida do paciente e de sua família 5 .

Além disso, a fadiga pode ser mais acentuada em pacientes com DRC em TRS, devido às longas distâncias e vias de difícil acesso percorridas para acessar os STRS e, ainda, por vezes, pela indisponibilidade de transporte, gerando cansaço físico e psicológico, devido ao fato de os STRS

A fadiga pode ser mais acentuada em pacientes com DRC em TRS, devido às longas distâncias e vias de difícil acesso percorridas para acessar os STRS e, ainda, por vezes, pela indisponibilidade de transporte, gerando cansaço físico e psicológico. estarem centralizados nos grandes centros econômicos dos estados ${ }^{6}$. Portanto, faz-se necessário olhar para esta temática, tendo em vista que a fadiga gera o impacto negativo na QV de pessoas com DRC em tratamento hemodialítico. Sendo assim, esse estudo objetivou avaliar a prevalência, intensidade e severidade do sintoma de fadiga em pessoas com Doença Renal Crônica em tratamento hemodialítico.

\section{Materiais e Método}

Estudo quantitativo descritivo, seguiu o checklist STROBE para estudos observacionais. Recorte da macropesquisa "Atenção à saúde nos serviços de terapia renal substitutiva da Metade Sul do Rio Grande do Sul" e "Empoderamento e autonomia para pessoas dos serviços de terapia renal da metade sul do Rio Grande do Sul". Realizado nos municípios de Pelotas, Rio Grande, São Lourenço do Sul, Alegrete e Uruguaiana, caracterizando a metade Sul do Rio Grande do Sul e 
Fronteira Oeste do Rio Grande do Sul e, também, por serem os municípios desta região os que disponibilizam o Serviço de Terapia Renal Substitutiva (STRS).

A amostra foi composta de 335 pacientes em tratamento hemolítico, dos 364 cadastrados nos STRS, 29 foram excluídos por ausência no serviço no momento da coleta ou por não terem condições de responder, no período de 2016 e 2017 e àqueles que participaram da macropesquisa. Foram critérios de seleção da amostra da macropesquisa, ser usuário cadastrado no serviço e estar presente no momento da coleta de dados; ter mais de 18 anos; capacidade de comunicar-se verbalmente; capacidade cognitiva preservada. Para a construção do banco de dados foi realizada dupla digitação no software EpiDATA 3.1, e, posteriormente, os dois bancos foram pareados e ajustadas as inconsistências, em seguida as variáveis foram analisadas com o software Stata 13.0.

Para mensurar a ocorrência de fadiga, utilizaram-se às variáveis da escala analógica visual Visual Analog Scale"-VAS de $100 \mathrm{~mm}^{7}$ e a escala de severidade de fadiga ${ }^{3}$ por meio de análise estatística descritiva, com distribuição de frequências absolutas e relativas e medidas de tendência central (médias e medianas) e de dispersão (desvio-padrão) das variáveis. Para identificar a intensidade e a severidade da fadiga, avaliou-se a intensidade de fadiga com a média e o desvio-padrão obtidos na variável escala analógica de fadiga. Já, para a severidade da fadiga, obteve-se um escore composto das respostas das nove questões que compõem a escala, a qual tem opções de resposta com notas que variam de 1 a 7 . O escore final obtido na escala variou de 9 a 63, sendo considerado um indicativo de escores de fadiga a partir de 28 . Resultados entre 28 e 39 caracterizam fadiga leve, de 40 a 51 moderada e de 52 a 63 grave $^{8}$.

Para cada variável analisada foi expresso o valor observado na amostra ( $\mathrm{n}$ ) e o percentual (\%). Adotou-se o $p$-valor $<0,05$ para assumir a hipótese de associação entre as variáveis sociodemográficas e clínicas com o sintoma de fadiga. Para tanto, foram comparadas as variáveis dependentes, escala analógica de fadiga e escala de severidade de fadiga e independentes, sexo, estado civil, idade, cor da pele, fonte de renda, renda, comorbidades, mora sozinho, anos de estudo e município pelo teste não paramétrico de Kruskal-Wallis e Mann-Whitney ${ }^{9}$.

Foram seguidos os princípios éticos da Resolução 466/2012 ${ }^{10}$ e recebeu aprovação do Comitê de Ética em Pesquisa da Faculdade de Enfermagem com o parecer número 1.386385 e CAAE 51678615300005316.

\section{Resultados}

Identificou-se a prevalência de $63,0 \%$ de fadiga entre os entrevistados com base nos resultados obtidos com os 335 participantes. A intensidade de fadiga verificada teve o valor médio de 42,3(DP=32,2) variando de 0 a 99, com mediana de 45. Quanto à severidade de fadiga, responderam a essa variável 303 dos 335 entrevistados, obtendo-se o valor médio de $35,8(\mathrm{DP}=17,8)$ e variando de 9 a 63 com mediana de 37.

As características sociodemográficas das pessoas em hemodiálise na metade sul do Rio Grande Sul e os escores de severidade do sintoma de fadiga são apresentados na tabela 1. 
Tabela 1 - Distribuição das características sociodemográficas e clínicas dos pacientes em hemodiálise na metade sul do Rio Grande do Sul pela severidade de fadiga, Pelotas, RS, 2017, $\mathrm{N}=303$.

\begin{tabular}{|c|c|c|c|c|c|c|c|c|c|c|c|}
\hline \multirow{3}{*}{$\begin{array}{l}\text { Características dos } \\
\text { entrevistados (n) }\end{array}$} & \multicolumn{11}{|c|}{ Severidade da fadiga } \\
\hline & \multicolumn{2}{|c|}{ Ausência } & \multicolumn{2}{|c|}{ Leve } & \multicolumn{2}{|c|}{ Moderada } & \multicolumn{2}{|c|}{ Severa } & \multicolumn{2}{|c|}{$\begin{array}{c}\text { Total } \\
\mathbf{N}=303\end{array}$} & \multirow[t]{2}{*}{$\begin{array}{l}\text { Valor } \\
\text { de } p\end{array}$} \\
\hline & $\mathbf{n}$ & $\%$ & $\mathbf{n}$ & $\%$ & $\mathbf{n}$ & $\%$ & $\mathbf{N}$ & $\%$ & $\mathbf{N}$ & $\%$ & \\
\hline \multicolumn{12}{|l|}{ Sexo (302) } \\
\hline Feminino & 40 & 31,5 & 26 & 20,5 & 26 & 20,5 & 35 & 27,6 & 127 & 42,0 & \multirow{2}{*}{$0,09^{*}$} \\
\hline Masculino & 71 & 40,6 & 32 & 18,3 & 36 & 20,6 & 36 & 20,6 & 175 & 58,0 & \\
\hline \multicolumn{12}{|l|}{ Estado civil (303) } \\
\hline Casado & 68 & 39,8 & 32 & 18,6 & 36 & 21,1 & 35 & 20,5 & 171 & 56,4 & \multirow{4}{*}{$0,19^{* *}$} \\
\hline Solteiro & 28 & 40,0 & 13 & 18,6 & 12 & 17,1 & 17 & 24,3 & 70 & 23,1 & \\
\hline Divorciado/Separado & 6 & 24,0 & 8 & 32,0 & 5 & 20,0 & 6 & 24,0 & 25 & 8,3 & \\
\hline Viúvo & 10 & 27,0 & 5 & 13,5 & 9 & 24,3 & 13 & 35,2 & 37 & 12,2 & \\
\hline \multicolumn{12}{|l|}{ Idade (299) } \\
\hline $18-19$ & 1 & 33,3 & 0 & 0 & 2 & 66,7 & 0 & 0 & 3 & 1,0 & \multirow{3}{*}{$0,27^{* *}$} \\
\hline $20-59$ & 66 & 42,3 & 26 & 16,7 & 32 & 20,5 & 32 & 20,5 & 156 & 52,2 & \\
\hline 60 a mais & 45 & 32,1 & 30 & 21,4 & 28 & 20,0 & 37 & 53,6 & 140 & 46,8 & \\
\hline \multicolumn{12}{|l|}{ Cor da pele (302) } \\
\hline Branco & 68 & 33,0 & 42 & 20,4 & 46 & 22,3 & 50 & 24,3 & 206 & 68,2 & \multirow{2}{*}{$0,08^{*}$} \\
\hline Não Branco & 44 & 45,8 & 16 & 16,7 & 15 & 15,6 & 21 & 21,9 & 96 & 31,8 & \\
\hline \multicolumn{12}{|l|}{ Fonte de renda (300) } \\
\hline Emprego & 2 & 22,2 & 2 & 22,2 & 2 & 22,2 & 3 & 33,4 & 9 & 3,0 & \multirow{4}{*}{$0,77^{* *}$} \\
\hline Renda familiar & 11 & 32,4 & 7 & 20,6 & 8 & 23,5 & 8 & 23,5 & 34 & 11,3 & \\
\hline Benefício & 35 & 38,9 & 16 & 17,8 & 17 & 18,9 & 22 & 24,4 & 90 & 30,0 & \\
\hline Aposentadoria & 63 & 37,7 & 33 & 19,8 & 33 & 19,8 & 38 & 22,7 & 167 & 55,7 & \\
\hline \multicolumn{12}{|l|}{ Renda (302) } \\
\hline Menor 880,00 & 17 & 37,8 & 3 & 6,6 & 8 & 17,8 & 17 & 37,8 & 45 & 14,9 & \multirow{4}{*}{$0,16^{* *}$} \\
\hline 880,01 a 1760,00 & 49 & 38,3 & 20 & 15,6 & 30 & 23,4 & 29 & 22,7 & 128 & 42,4 & \\
\hline 1760,01 a 2640,00 & 27 & 32,5 & 19 & 22,9 & 17 & 20,5 & 20 & 24,1 & 83 & 27,5 & \\
\hline Maior que 2,640,01 & 19 & 41,3 & 15 & 32,6 & 7 & 15,2 & 5 & 10,9 & 46 & 15,2 & \\
\hline Comorbidades (291) & & & & & & & & & & & \\
\hline Nenhuma & 36 & 38,7 & 26 & 28,0 & 17 & 18,3 & 14 & 15,0 & 93 & 32,0 & \\
\hline $\mathrm{HAS}^{\&}$ & 44 & 37,0 & 17 & 14,3 & 26 & 21,8 & 32 & 26,9 & 119 & 40,9 & $0,16^{* *}$ \\
\hline $\mathrm{DM}^{\#}$ & 14 & 50,0 & 3 & 10,7 & 5 & 17,9 & 6 & 21,4 & 28 & 10,0 & \\
\hline HAS e DM & 15 & 29,4 & 9 & 17,6 & 13 & 25,5 & 14 & 27,5 & 51 & 17,5 & \\
\hline Mora sozinho (303) & & & & & & & & & & & \\
\hline Não & 101 & 38,1 & 52 & 19,6 & 51 & 19,3 & 61 & 23,0 & 265 & 87,5 & $0,22 *$ \\
\hline Sim & 11 & 28,9 & 6 & 15,8 & 11 & 28,9 & 10 & 26,4 & 38 & 12,5 & \\
\hline Anos de estudo (303) & & & & & & & & & & & \\
\hline Nãœstudou & 5 & 29,4 & 7 & 41,2 & 2 & 11,8 & 3 & 17,6 & 17 & 5,6 & \\
\hline Até 9 & 77 & 37,0 & 37 & 17,8 & 46 & 22,1 & 48 & 23,1 & 208 & 68,6 & $0,95^{* *}$ \\
\hline 10-16 & 30 & 38,5 & 14 & 18,0 & 14 & 18,0 & 20 & 25,6 & 78 & 25,7 & \\
\hline Município (302) & & & & & & & & & & & \\
\hline Pelotas & 40 & 30,1 & 26 & 19,5 & 33 & 24,8 & 34 & 25,6 & 133 & 44,0 & \\
\hline Rio Grande & 23 & 43,4 & 7 & 13,2 & 8 & 15,1 & 15 & 28,3 & 53 & 17,5 & \\
\hline São Lourenço do Sul & 7 & 26,9 & 7 & 26,9 & 4 & 15,4 & 8 & 30,8 & 26 & 8,6 & $0,10^{* *}$ \\
\hline Alegrete & 27 & 51,9 & 8 & 15,4 & 7 & 13,5 & 10 & 19,2 & 52 & 17,2 & \\
\hline Uruguaiana & 14 & 36,9 & 10 & 26,3 & 10 & 26,3 & 4 & 10,5 & 38 & 12,6 & \\
\hline
\end{tabular}

\&HAS (Hipertensão Arterial Sistêmica) * DM (Diabetes Melitus) *Teste de Mann-Whitney; **Teste de Kurskalwallis *** salário mínimo brasileiro em 2016

Fonte: Elaboração própria a partir do banco de dados dos projetos "Atenção à saúde nos serviços de terapia renal substitutiva da Metade Sul do Rio Grande do Sul" e "Empoderamento e autonomia para pessoas dos serviços de terapia renal da metade sul do Rio Grande do Sul". 
Dentre os participantes, 58,0\% $(n=175)$ eram do sexo masculino, porém, a maior concentração de fadiga ocorreu entre as mulheres $68,6 \%(n=87)$, sendo que $27,6 \% \quad(n=35)$ apresentaram fadiga severa. Já, em relação ao estado civil, com 303 entrevistados o predomínio foi de pessoas casadas com $56,4 \%(n=171)$, destas 39,8\% ( $n=68)$ não apresentavam fadiga e $41,6 \%(n=71)$ apresentavam fadiga entre moderada $21,1 \%(n=36)$ e severa $20,5 \%(n=35), 40,0 \%(n=70)$ eram solteiros e sem fadiga, entre divorciados ou separados $32,0 \%(n=25)$ houve fadiga leve e $35,2 \%(n=37)$ dos viúvos apresentaram fadiga severa. Em relação à idade $(n=299), 52,2 \%(n=156)$ tinham entre 2059 anos, sendo que $42,3 \%(n=66)$ sem fadiga e $41,0 \%(n=64)$ apresentaram fadiga, distribuindose igualmente entre moderada e severa. Já, entre os participantes com mais de 60 anos, $53,6 \%(n=37)$ apresentaram fadiga severa.

A cor da pele $(n=302)$ prevalente foi a branca com $68,2 \%(n=206)$ e $67 \%(n=138)$ destes apresentaram fadiga entre leve, moderada e severa. Dos $31,8 \%(n=96)$ não brancos, 45,8\%( $n=44)$ não apresentaram fadiga. A fonte de renda destas pessoas (300) concentra-se principalmente na aposentadoria por idade 55,7\%(167) e destes, 62,3\%(104) apresentam fadiga entre leve, moderada e severa. A renda (302) é para 42,4\%(128) de 880,01 a 1760,00 e destes, 61,7\%(79) apresentam fadiga, sendo $46,1 \%(59)$ entre fadiga moderada $23,4 \%(30)$ e severa $22,7 \%(29)$.

Quantoàs comorbidades $(n=291), 40,9 \%(n=119)$ dos participantes apresentaram Hipertensão Arterial Sistêmica, dos quais $63 \%(n=75)$ apresentavam fadiga, sendo $26,9 \%(n=32)$ severa.

\section{Quanto às comorbidades $(n=291)$, $40,9 \%(n=119)$ dos participantes apresentaram Hipertensão Arterial Sistêmica, dos quais $63 \%(n=75)$ apresentavam fadiga, sendo 26,9 $\%(n=32)$ severa.}

Possuíam companhia em sua moradia $87,5 \%(n=265)$ dos participantes, dos quais 61,9 $\%(n=164)$ apresentaram fadiga, sendo severa em $23 \%(n=61)$ dos casos. Já dos $12,5 \%(n=38)$ que moravam sozinhos, $71,1 \%(n=27)$ apresentaram fadiga e destes, $28,9 \%(n=11)$ com fadiga moderada. Os participantes apresentavam escolaridade baixa, até nove anos $68,6 \%(n=208)$ e destes, 63\%(n=131) apresentaram fadiga, sendo a severa a mais prevalente com $23,1 \%(n=48)$. Dentre os que tiveram escolaridade acima de dez anos, 61,6\%(n=44) apresentaram fadiga, sendo $25,6 \%(n=20)$ com fadiga severa, e este grupo também foi o que apresentou a maior porcentagem de pessoas com ausência de fadiga $38,5 \%(n=30)$.

Em relação ao município, os participantes foram oriundos de Pelotas em $44 \%(n=133)$ e destes, $69,9 \%(n=93)$ apresentaram fadiga, concentrando-se entre moderada $24,8 \%(n=33)$ e severa $25,6 \%(n=34)$. Outros municípios que demonstraram igualdade quase completa em quantidade de participantes foram Rio Grande 17,5\%(n=53) e Alegrete com 17,2\%(n=52), no entanto, entre eles existe uma diferença, pois, enquanto em Rio Grande, 56,6\%(n=30) apresentaram fadiga, sendo severa em 28,3\%(n=15), em Alegrete, 51,9\%(n=27) não demonstraram fadiga. Já, em São Lourenço e Uruguaiana que são serviços com menor número de participantes, ambos apresentaram altos índices de fadiga, em São Lourenço, 8,6\%(n=26) participantes apresentaram $73,1 \%$ de fadiga, sendo severa em $30,8 \%(n=8)$ e Uruguaiana com $12,6 \%(n=38)$ participantes, $63,1 \%(n=24)$ apresentaram fadiga, entre leve e moderada, distribuindo-se igualmente com $26,3 \%(n=10)$ cada um deles.

Ao analisar todas as variáveis, nenhuma teve significância estatística na distribuição pela severidade de fadiga. 


\section{Discussão}

Diante da subjetividade do sintoma de fadiga e do impacto que este causa sobre a qualidade de vida e saúde da pessoa em tratamento hemodialítico, nota-se a importância de identificar tal sintoma entre pessoas com DRC, posto que a existência do sintoma de fadiga foi encontrada em alguns estudos ${ }^{5,11}$ nas diferentes modalidades de terapia renal de substituição. Neste estudo, identificou-se existência de fadiga nos participantes, assim como em estudos internacionais que também encontraram alta existência deste sintoma, variando de $44,7 \%{ }^{12}$ a $100 \%{ }^{13}$ dos participantes.

A principal comorbidade encontrada neste estudo associadaà DRC equeinfluencianosintomadefadiga em pessoas fazendo tratamento hemodialítico foi a Hipertensão Arterial Sistêmica (HAS), sendo dentre os fadigados a maior prevalência com fadiga severa,

A principal comorbidade encontrada neste estudo associada à DRC e que influencia no sintoma de fadiga em pessoas fazendo tratamento hemodialítico foi a Hipertensão Arterial Sistêmica (HAS). o que corrobora os achados do estudo que elucidou a questão de que a HAS e Diabetes Mellitus (DM) são as principais doenças de base da DRC no Brasil $^{2}$, sendo responsáveis por quase metade dos indivíduos que estão em tratamento dialítico, sendo o DM a principal etiologia de base para a $\mathrm{DRC}^{13}$; já as comorbidades que influenciam diretamente na fadiga ${ }^{14}$ são os diagnósticos de anemia ${ }^{15}$, ansiedade e depressão ${ }^{5}$.

O gênero é outro fator que exerce influência no sintoma de fadiga, para o qual as mulheres são mais acometidas ${ }^{16}$, o que corrobora este estudo, o qual identificou um maior número de homens, embora a concentração maior fadiga apresentou-se nas mulheres. Considera-se que essa porcentagem de mulheres esteja associada à função hormonal e, muitas vezes, pela dupla jornada de trabalho, com emprego formal, cuidado doméstico, cuidado com filhos, além do tratamento são alguns fatores que possivelmente justifique este achado. Da mesma forma, pessoas de etnia branca são mais propensas a desenvolver o sintoma de fadiga, o que pode estar relacionado com a maior quantidade de pessoas brancas em tratamento hemodialítico, uma vez que no estado do Rio Grande do Sul a predominância da população é de cor branca ${ }^{17}$. Embora esperava-se encontrar uma maior quantidade de negros, pois possuem maior probabilidade de desenvolver hipertensão e diabetes ${ }^{18}$, que são preditivos de DRC.

Evidenciou-se neste estudo que a principal fonte de renda foi a aposentadoria por idade em que os participantes ganhavam entre $R \$ 880,01$ a $R \$ 1760,00$ equivalentes a aproximadamente $\$ 220,00$ a $\$ 440,00$ na época da coleta do estudo, a renda concentrava-se entre um e dois salários mínimos, o que também pode ser relacionado com o comprometimento das atividades do trabalho, devido às limitações e ao tempo gasto para a realização das modalidades terapêuticas (hemodiálise), propiciando o desemprego e, assim, um maiorimpacto socioeconômico. Osfatores socioeconômicos foram referidos no estudo que apontou o baixo nível de escolaridade como o principal fator que influencia na renda e adesão ao tratamento ${ }^{16}$, o que está em conformidade com este estudo, em que os participantes possuíam baixo índice de escolaridade, com até 9 anos de estudo. O grau de escolaridade, também, se destaca na promoção de saúde, pois a partir dele pode-se avaliar a qualidade das informações prestadas e o nível de compreensão demonstrado pelo paciente em relação às recomendações feitas pelo profissional da saúde, sobretudo no que se refere aos cuidados em saúde, hábitos de vida e o tratamento ${ }^{19}$. Quanto maior a escolaridade, maior é o acesso às informações e melhoria das condições econômicas, 
desse modo, pessoas com maior grau de escolaridade exercem atividades que exigem menor esforço físico, e por isso o impacto da doença em suas atividades é menor, além de assimilar mais adequadamente as informações e colaborar com a adesão ao tratamento ${ }^{19}$.

Identificou-se que pessoas que não moram sozinhas estão em maior número dos participantes, porém as que moram sozinhas foram as mais fadigadas, prevalecendo entre moderada e severa, indo ao encontro do estudo que revelou que baixo apoio familiar piorou o sintoma de fadiga, enquanto um alto nível de apoio social diminuiu o nível de fadiga ${ }^{14}$. Assim como o número de pessoas na residência demonstrou ser fator auxiliar, pois a existência de um companheiro e/ou residir com a família pode contribuir para a manutenção dos cuidados no domicílio, bem como nas atividades diárias. O que foi evidenciado neste estudo em que os casados representaram menor índice de fadiga, portanto, o estado civil tem influência no sintoma de fadiga, pois ter um companheiro melhora o enfrentamento das adversidades, tal e qual o apoio familiar e de amigos age como alavanca para manter o equilíbrio, estimula as mudanças dos hábitos e promove determinados comportamentos que melhoram,

O que foi evidenciado neste estudo em que os casados representaram menor índice de fadiga, portanto, o estado civil tem influência no sintoma de fadiga, pois ter um companheiro melhora o enfrentamento das adversidades. por sua vez, a saúde geral e a qualidade de vida ${ }^{20}$.

Evidenciou-se também por meio deste estudo, que os viúvos e as pessoas com mais de 60 anos apresentaram fadiga severa, fator possivelmente relacionado com a falta do companheiro/a e a necessidade do apoio da família no caso dos idosos, pois eles tendem a ser mais dependentes para a realização das atividades diárias, portanto, o apoio familiar impacta em melhor qualidade de vida dessas pessoas ${ }^{4,14,18,21}$.

$\mathrm{Na}$ variável relacionada com o município, identificou-se que praticamente metade dos participantes do estudo realizavam TRS em Pelotas, e com maior índice de fadiga, possivelmente pelo fato de Pelotas ser uma cidade de grande porte na metade sul do Rio Grande do Sul, posto que em municípios de médio e grande porte, a quantidade e intensidade dos laços afetivos são menores (ou muitas vezes inexistentes), diferentemente do que acontece em cidades pequenas, onde a intensidade dos relacionamentos interpessoais são bem maiores ${ }^{22}$. Além de fatores como violência, falta de segurança, trânsito e outros estressores que propiciam o isolamento social e o desencadeamento de transtornos de humor nas grandes cidades e nos ambientes agitados das grandes metrópoles. Evidenciamos isto em relação ao município de Alegrete em que a maioria dos participantes não apresentaram o sintoma de fadiga, o que, também, pode estar relacionado com a cultura local, o tamanho da cidade e o Índice de Desenvolvimento Humano (IDHM) da cidade, pois, em 2010, o IDHM de Alegrete era de 0,740, o que situa o referido município na faixa de Desenvolvimento Humano Alto (IDHM entre 0,700 e 0,799). A dimensão que mais contribui para o IDHM do município é Longevidade, com índice de 0,849 , seguida de Renda, com índice de 0,720, e de Educação, com índice de 0,664²3.

Os serviços de hemodiálise de Pelotas ainda possuem outro fator que influencia o alto índice de fadiga, uma vez que recebe pacientes advindos de outros municípios da região. O município mais distante de Pelotas fica localizado a 250 km, com duração em média de viagem as cidades de três horas, afetando a qualidade de vida das pessoas que necessitam percorrer longas distâncias, com transporte precário, gerando desgaste físico e emocional para receber o tratamento hemodialítico ${ }^{6}$. Tratamento que possibilita a melhoria dos sintomas, porém esse recurso é ininterrupto, tornando-se cansativo e repetitivo, uma vez que a rotina contínua e duradoura 
das sessões impõe ao paciente a interrupção das suas atividades diárias, a dedicação de quatro horas, três vezes por semana, diante disto, as intervenções lúdicas, se bem conduzidas, podem ser satisfatoriamente aceitas e aproveitadas em casos de tratamento duradouro, tornam-se aliadas para suavizar o peso do processo crônico, pois é durante as sessões de hemodiálise que as pessoas com DRC compartilham um período de convivência e de diálogo, uma oportunidade para expressar emoções e compartilhar medos e temores com outros pacientes e profissionais da saúde, favorecida pelas ações de promoção da saúde, melhorando a auto-estima, depressão e necessidade de convívio social, o que pode impactar positivamente na qualidade de vida destes ${ }^{24}$.

Além das atividades lúdicas favorecerem as ações de promoção da saúde, podemos também mencionar o uso da tecnologia como um potencial de integração inteligente entre os serviços de saúde e a continuidade dos cuidados, aumentando o espectro de possibilidades de atuação e alcançando, simultaneamente, um número incontável de pessoas ${ }^{25}$ permitido um autocuidado mais eficaz, assim proporcionando melhor qualidade de vida as pessoas em tratamento hemodialítico.

\section{Conclusão}

Baseando-se na análise dos dados concluiu-se que $63 \%$ dos participantes apresentavam o sintoma de fadiga, com intensidade variando de 0 a 99 e mediana de 45, já a severidade variando de 9 a 63, com mediana de 37. Ao correlacionar com aspectos sociodemográficos e clínicos, evidenciou-se que as mulheres, viúvos, idosos e hipertensos apresentaram maior predomínio de fadiga severa e que o fator psicossocial e socioeconômico influencia no sintoma de fadiga, assim como o apoio da família e o fato de não morar sozinho pode aumentar a qualidade de vida de pessoas em tratamento hemodialítico.

As ações de promoção de saúde favorecem o entendimento da doença, proporcionam momentos de troca e permitem aos profissionais da saúde conhecer melhor o perfil da clientela atendida para o planejamento de cuidados visando uma assistência de qualidade. Assim enfermeiros, como a equipe multiprofissional que atuam na atenção a pessoa com DRC, devem procurar identificar e minimizar os sintomas de fadiga, avaliando seus exames laboratoriais, fazendo o diagnóstico precoce da fadiga, por meio da aplicação das escalas disponíveis para tal, com efeitos simples e de baixo custo. Da mesma forma podem fazer uso do genograma e ecomapa, para conhecer a sua rede de apoio social e de apoio, promovendo maior qualidade de vida, melhorando seu autocuidado e tarefas do dia a dia e, consequentemente, aumentando sua autoestima e o bem-estar.

O estudo tem limitações de regionalidade, por ter sido aplicado em uma área especifica do Rio Grande do Sul, sendo assim, os resultados podem ser diferentes em demais regiões do Brasil.

Conflito de interesses: Os autores declaram que não há conflitos de interesse.

Financiamento: O estudo recebeu financiamento do CNPq por meio do processo 442502/20141 e PQ 2016-Chamada CNPq N $\circ$ 12/2016. 


\section{Referências}

1. KidneyDisease: Improving Global Outcomes (KDIGO) CKD WorkGroup. KDIGO 2012 clinicalpracticeguideline for theevaluationand management ofchronickidneydisease. KidneyIntSuppl. 2013;3(1):1-150. https://kdigo.org/wp-content/uploads/2017/02/KDIGO_2012_CKD_GL.pdf

2. Neves PDMM, Sesso RCC, Thomé FS, Lugon JR, Nascimento MM. Censo Brasileiro de Diálise: análise de dados da década 2009-2018. Braz. J. Nephrol. 2020;42(2):191-200. https://doi.org/10.1590/2175-8239-JBN-2019-0234

3. Krupp LB, Larocca NG, Muir-Nash J, Steinberg AD. The Fatigue SeverityScaleApplicationtoPatientsWithMultipleSclerosisandSystemicLupusErythematosus. ArchNeurol. 1989;46(10):1121-123. https://doi.org/10.1001/archneur.1989.00520460115022

4. Bossola M, Stasio ED, Antocicco M, Panico L, Pepe G, Tazza L. Fatigue Is Associated withIncreased Risk ofMortality in PatientsonChronicHemodialysis. Nephron. 2015;130(2):113118. https://doi.org/10.1159/000430827

5. Bossola M, Di Stasio E, Marzetti E, De Lorenzis K, Pepe G, Vulpio C. Fatigue is associated with high prevalence and severity of physical and emotional symptoms in patients on chronic hemodialysis. Int Urol Nephrol. 2018; 50(7): 1341-1346.

https://doi.org/10.1007/s11255-018-1875-0

6. Schwartz E, Lise F, Santos BP, Garcia RP,Dall"Agnol J, Rodrigues LPV, Soares Farias JS. Caracterização dos serviços de terapia renal substitutiva da metade sul do Rio Grande do Sul/Brasil. Enfermagem Revista. 2017;20(2):86-98. file://D:/_Datos\%20de\%20Usuario/ Descargas/16325-Texto\%20do\%20artigo-57508-1-10-20171026.pdf

7. Hewlett SE, Dures C, Almeida C. Measuresof fatigue: Bristol RheumatoidArthritis Fatigue Multi-Dimensional Questionnaire (BRAFMDQ), Bristol RheumatoidArthritis Fatigue Numerical Rating Scales (BRAF NRS) for severity, effect, andcoping, Chalder Fatigue Questionnaire (CFQ), Checklist. ArthritisCare Res (Hoboken). 2011;63 (S11):S263-S286.

https://doi.org/10.1002/acr.20579

8. Valderramas S, Feres AC, Melo A. Reliability and validity study of a Brazilian-Portuguese version of the fatigue severity scale in Parkinson's disease patients. Arq. Neuro-Psiquiatr.2012; 70(7): 497-500. https://doi.org/10.1590/S0004-282X2012000700005

9. Hulley SB, Cummings SR, Browner WS, Grady D, Hearst N, Newman TB. Outlining clinical research: anepidemiological approach. $2^{\text {nd }}$ ed. Porto Alegre(RS): Artmed; 2003.

10.Brasil. Conselho Nacional de Saúde. Resolução n 466, de 12 de dezembro de 2012. Diário Oficial da União, Poder Executivo, Brasília, DF, 13 jun. 2013. Seção 1, n. 112, p. 59-62.

https://conselho.saude.gov.br/resolucoes/2012/Reso466.pdf

11.Jhamb M, Liang K, Yabes J, Steel J, Dew MA, Niray S, et al. Prevalence and Correlates of Fatigue in CKD and ESRD: Are Sleep Disorders a Key to Understanding Fatigue?. Am J Nephrol. 2013; 38(6):489-495. https://doi.org/10.1159/000356939

12.Joshwa B, Khakha CD, Mahajan S. Fatigue and Depression and Sleep Problems among Hemodialysis Patients in a Tertiary Care Center. Saudi J KidneyDisTranspl. 2012;23(4):729-735. https://doi.org/10.4103/1319-2442.98149

13.Biniaz V, Tayybi A, Nemati E, Sadeghi Shermeh M, Ebadi A. Different aspects of fatigue experienced by patients receiving maintenance dialysis in hemodialysis units. Nephrourol Mon. 2013;5(4):897-900. https://doi.org/10.5812/numonthly.11667

14.Wang SY,Zang XY, Fu SH,Bai J, Liu JD,Tian L,Feng YY,Zhao Y. Factors related to fatigue in Chinese patients with end-stage renal disease receiving maintenance hemodialysis: a multicenter cross-sectional study. RenFail. 2016;38 (3):442-450.

https://doi.org/10.3109/0886022X.2016.1138819 
15.Picariello F, Moss-Morris R, Macdougall, I.C, Norton S, Da Silva-Gane M, Farrington K, Clayton H, Chilcot J. Cognitive-behaviouraltherapy (CBT) for renal fatigue (BReF): a feasibilityrandomised-controlledtrialof CBT for the management of fatigue in haemodialysis (HD) patients. BMJ Open. 2018;8 (3):e020842. https://doi.org/10.1136/bmjopen-2017-020842

16.Francisco PMSB, Segri NJ, Borim FSA, Malta DC. Prevalência simultânea de hipertensão e diabetes em idosos brasileiros: desigualdades individuais e contextuais, Ciênc. Saúde Colet. 2018;23(11):3829-3840. https://doi.org/10.1590/1413-812320182311.29662016

17.Instituto Brasileiro de Geografia e Estatística (IBGE). Censo. Amostra- Caracteristicas da população. Río Grande del Sur. 2010. https://cidades.ibge.gov.br/brasil/rs/pesquisa/23/25888

18. Yang PC, Lu YY. Predictors of Fatigue among Female Patients on Hemodialysis. NephrolNurs J. 2017; 44(6):533-539. https://go.gale.com/ps/i.do?p=AONE\&u=anon 7f9e1817\&id=GALE $A 523213260 \& v=2.1 \& i t=r \& s i d=$ googleScholar\&asid=03399cb2

19.Marinho CLA, Oliveira JF, Borges JES, Silva RS, Fernandes FECV. Quality of life of chronic renal patients undergoing hemodialysis. Rev Rene. 2017;18(3):396-403.

https://doi.org/10.15253/2175-6783.2017000300016

20.Silva KG, Freitas BA, Furgêncio GK, Portes LA, Kutz NA, Salgueiro MMHAO. Relação entre a qualidade de vida e o consumo alimentar de professores de rede privada. Rev. pesqui. cuid. Fundam. 2017;9(4):962-970. https://doi.org/10.9789/2175-5361.2017.v9i4.962-970

21.Bossola M, Di Stasio E, Sirolli V, Ippoliti F, Cenerelli S, Monteburini T, Parodi E, Santarelli S, Nebiolo PE, Bonomini M, Picca A, Calvani R, Marzetti E. Prevalence and Severity of Pos tdialysis Fatigue Are Higher in Patients on Chronic Hemodialysis With Functional Disability .Ther Apher Dial. 2018;22(6):635-640. https://doi.org/10.1111/1744-9987.12705

22.Negri EC, Sampaio ACLS, Silva ACP, Paulo HM, Costa LB, Souza NFH. Qualidade de vida do paciente com insuficiência renal crônica submetido à hemodiálise. Colloq Vitae. 2016;8(2):3236. https://doi.org/10.5747/cv.2016.v08.n2.v165

23.ADHB. Alegrete, RS. Atlas de Desenvolvimento Humano do Brasil. http://www.atlasbrasil.org.br/perfil/municipio/430040 Acesso: 16 fev. 2021.

24.Paula TB, Souza BM, Medeiro N, Malt S, Mouhssen El, GutierrezF, Lourenço LA,Zihlmann KF. Potencialidade do Lúdico como Promoção de Bem-Estar Psicológico de Pacientes em Hemodiálise. Psicologia: Ciência e Profissão. 2017;37(1): 146-158.

https://doi.org/10.1590/1982-3703000682014

25. Oliveira JGR, Silva Júnior GB, Vasconcelos Filho JE. Doença Renal Crônica: Explorando novas estratégias de comunicação para promoção de saúde. Revista Brasileira de Promoção da Saúde. 2017;31(4):1-8. https://doi.org/10.5020/18061230.2018.8753 